\title{
On the interpretation of the enthalpy of solvation of hydroxyl cyclohexane derivatives in different solvents
}

\author{
Felisbela S. Costa,
}

Laboratório de Métodos Instrumentais de Análise, Faculdade de Farmácia, Universidade de Coimbra, Coimbra, Portugal

M. Ermelinda Eusébio, J. S. Redinha, and M. Luísa P. Leitão ${ }^{a}$

Departamento de Química, Universidade de Coimbra, Coimbra, Portugal

\begin{abstract}
Scaled particle theory was used for calculating the enthalpy of cavity formation in the solvation process of hydroxyl cyclohexane derivatives in water and in organic solvents. From the values calculated for the enthalpy of formation of the cavity and from those quoted for the enthalpy of solvation, the enthalpy of solute/solvent interaction was determined.

This thermodynamic property shows that the formation of a cavity is an important contribution to the enthalpy of solvation, and is strongly dependent on the structure of the solvent. Unexpected results observed for water as compared with organic solvents are due to the differences in the cavity formation term rather than to solute/solvent interactions. (c) 2000 Academic Press
\end{abstract}

KEYWORDS: solvation enthalpy; non-electrolyte solutions; hydroxyl cyclohexane derivatives

\section{Introduction}

The enthalpy of solvation is a very useful property for studying solutions. On one hand the enthalpy is a very sensitive property to structural variations in solution, and, on the other, as intermolecular forces in the initial state are practically non-existent, the thermodynamic property is closely related to the effect produced in the solvent by the introduction of a solute molecule.

It is important to note that the thermodynamic properties related to a solvation process do not depend exclusively, even for very dilute solutions, on solute/solvent interaction because the addition of a solute involves the formation of a cavity of adequate size to hold

${ }^{a}$ To whom correspondence should be addressed (E-mail: mlleitao@cygnus.ci.uc.pt). 
the solute molecule. The disruption of the structure of the solvent requires an amount of energy which depends on the solute molecule size and the solvent structure. For structured solvents cavity formation is always a significant term, and the thermodynamic properties of solvation may lead to incorrect conclusions if the cavity term is not taken into account. Hence any experimental effort to determine the effect produced on a solvent by adding a solute enhances the importance of thermodynamics in the interpretation of solvation.

Following a classic scheme, dissolution can be considered as a two-step process: (1) formation of a cavity in the solvent to hold the solute molecule; and (2) introduction of the solute into the cavity and switching on of the interaction forces between the solute and the solvent. This method of interpretation of the solvation process goes back to the 1930s. ${ }^{(1,2)}$ Since then progress has been made resulting in better theories that can be used for calculating the term corresponding to the cavity formation. One of the theories used for this purpose is the scaled particle theory (SPT) developed in 1959 by Reiss et $a l .{ }^{(3)}$ for hard sphere liquids, and refined later in various papers. ${ }^{(4)}$ Pierotti succeeded in applying the SPT to the study of non-polar gases in real solvents including water. ${ }^{(5-7)}$

Since then the theory has been used for calculating the cavity term of different thermodynamic properties of solvation. Although SPT was developed for hard sphere fluids it is nevertheless applicable to real liquids because it contains solute and solvent structural features which fit the equations for a wide range of systems. It is, therefore, a semi-empirical theory, but constitutes the most powerful method for calculating the cavity term in the solvation process. Comments on the application of the theory can be found in the literature. ${ }^{(8-14)}$ Other methods have been proposed for calculating the contribution of the cavity formation. Their results differ significantly from those of the SPT according to some authors, ${ }^{(15)}$ but agree satisfactorily with those of the SPT according to others. ${ }^{(16)}$

In the present work the enthalpy of solute/solvent interaction is calculated from the enthalpy of solvation and from the enthalpy of cavity creation, this last quantity being calculated using the initial version of SPT. The systems to which the method was applied are solutions constituted by cyclohexane, cyclohexanol, cis-1,2-cyclohexanediol, and myoinositol as solutes and water (W), formamide (FMD), and dimethylsulphoxide (DMSO) as solvents.

\section{Determination of the enthalpy of solute/solvent interaction}

The enthalpies of solvation at infinite dilution for the systems under study were determined by the present authors, ${ }^{(17)}$ except those involving cyclohexane which were taken from the literature. ${ }^{(18)}$ Cyclohexane gives information on the interaction of the non-polar part of the solute with the solvents which is useful to incorporate into the discussion of the data for compounds of similar molecular structure but with different numbers of $\mathrm{OH}$ groups. The results for the enthalpy of solvation at infinite dilution $\Delta_{\text {solv }} H_{\mathrm{m}}^{\infty}$ of the solutes in the different solvents are given in table 1.

Pierotti ${ }^{(7)}$ proposed an equation that relates the enthalpy of solvation $\Delta_{\text {solv }} H_{\mathrm{m}}^{\infty}$ to the enthalpy of cavity formation $\Delta_{\text {cav }} H$ and the enthalpy of solute/solvent interaction $\Delta_{\text {int }} H_{\mathrm{m}}^{\infty}$ as follows:

$$
\Delta_{\text {solv }} H_{\mathrm{m}}^{\infty}=\Delta_{\mathrm{cav}} H+\Delta_{\mathrm{int}} H_{\mathrm{m}}^{\infty}-R T+\alpha R T^{2},
$$


TABLE 1 . Enthalpy of solvation $\Delta_{\mathrm{solv}} H_{\mathrm{m}}^{\infty}$ for hydroxyl cyclohexane derivatives in different solvents at $T=298.15 \mathrm{~K}$

\begin{tabular}{lcccc}
\hline Solvent & Cyclohexane & $\begin{array}{c}\text { Cyclohexanol }^{d} \\
\Delta_{\text {solv }} H_{\mathrm{m}}^{\infty} /\left(\mathrm{kJ} \cdot \mathrm{mol}^{-1}\right)\end{array}$ & \\
\hline $\mathrm{W}$ & $-33.2^{a}$ & -70.7 & -82.4 & -165.8 \\
FMD & $-25.9^{b}$ & -57.2 & -70.3 & -166.6 \\
DMSO & $-21.6^{c}$ & -55.9 & -69.7 & -169.7 \\
\hline
\end{tabular}

${ }^{a}$ Reference $18 \mathrm{a} ;{ }^{b}$ reference $18 \mathrm{~b} ;{ }^{c}$ reference $18 \mathrm{c} ;{ }^{d}$ reference 17.

TABLE 2. Solvent molecular diameter $\sigma_{1}$, molar volume $V_{1}^{*}$, and cubic expansion coefficient $\alpha$ at $T=298.15 \mathrm{~K}$

\begin{tabular}{lccc}
\hline Solvent & $\frac{\sigma_{1}}{\mathrm{~nm}}$ & $\frac{V_{1}^{* d}}{\mathrm{~cm}^{3} \cdot \mathrm{mol}^{-1}}$ & $\frac{\alpha \cdot 10^{3 d}}{\mathrm{~K}^{-1}}$ \\
\hline $\mathrm{W}$ & $0.275^{a}$ & 18.07 & 0.257 \\
$\mathrm{FMD}$ & $0.394^{b}$ & 39.9 & 0.746 \\
DMSO & $0.491^{c}$ & 71.3 & 0.982 \\
\hline
\end{tabular}

${ }^{a}$ References $6,19,20 ; \quad b$ reference 12 ; ${ }^{c}$ references 21,$22 ; \stackrel{d}{d}$ reference 23 .

where $\alpha$ is the cubic expansion coefficient of the solvent, $R$ the ideal gas constant, and $T$ the temperature.

The enthalpy for creating a cavity whose radius $r=1 / 2\left(\sigma_{1}+\sigma_{2}\right)$ is calculated from:

$\Delta_{\mathrm{cav}} H=\alpha R T^{2} \frac{y}{(1-y)^{3}}\left\{(1-y)^{2}+3(1-y)\left(\frac{\sigma_{2}}{\sigma_{1}}\right)+3(1+2 y)\left(\frac{\sigma_{2}}{\sigma_{1}}\right)^{2}\right\}+p V_{1}^{*} y\left(\frac{\sigma_{2}}{\sigma_{1}}\right)^{3}$,

where $\sigma_{1}$ and $\sigma_{2}$ are the solvent and solute molecular diameters, $p$ the pressure, $y=$ $N_{\mathrm{A}} \pi \sigma_{1}^{3} /\left(6 V_{1}^{*}\right)$, in which $V_{1}^{*}$ is the molar volume of the solvent and $N_{\mathrm{A}}$ is the Avogadro number. The parameter $y$ is a quantity that gives an indication of the molar volume fraction occupied by the molecules.

The values used for the properties of the solvents in the calculation of $\Delta_{\text {cav }} H$ are listed in table 2. The molecular diameters of the solute were calculated from the van der Waals volumes obtained from Bondi molecular group increments, and are as follows: cyclohexane, $\sigma_{2}=0.572 \mathrm{~nm}$; cyclohexanol, $\sigma_{2}=0.586 \mathrm{~nm}$; cis-1,2cyclohexanediol, $\sigma_{2}=0.600 \mathrm{~nm}$; and $m y o$-inositol, $\sigma_{2}=0.650 \mathrm{~nm} .^{(24)}$

Table 3 contains the results obtained for $\Delta_{\text {cav }} H$ and $\Delta_{\text {int }} H_{\mathrm{m}}^{\infty}$ for the systems under study. 
TABLE 3. Values calculated for the enthalpies of cavity formation $\Delta_{\text {cav }} H$ and solute/solvent interaction $\Delta_{\mathrm{int}} H_{\mathrm{m}}^{\infty}$ for hydroxyl cyclohexane derivatives in several solvents at $T=298.15 \mathrm{~K}$

\begin{tabular}{lcccccccc}
\hline & \multicolumn{2}{c}{ Cyclohexane } & \multicolumn{2}{c}{ Cyclohexanol } & \multicolumn{2}{c}{$c i s-1,2-C y c l o h e x a n e d i o l$} & \multicolumn{2}{c}{$m y o-$ Inositol } \\
\multirow{2}{*}{ Solvent } & $\frac{\Delta_{\mathrm{cav}} H}{\mathrm{~kJ} \cdot \mathrm{mol}^{-1}}$ & $\frac{\Delta_{\mathrm{int}} H_{\mathrm{m}}^{\infty}}{\mathrm{kJ} \cdot \mathrm{mol}^{-1}}$ & $\frac{\Delta_{\mathrm{cav}} H}{\mathrm{~kJ} \cdot \mathrm{mol}^{-1}}$ & $\frac{\Delta_{\mathrm{int}} H_{\mathrm{m}}^{\infty}}{\mathrm{kJ} \cdot \mathrm{mol}^{-1}}$ & $\frac{\Delta_{\mathrm{cav}} H}{\mathrm{~kJ} \cdot \mathrm{mol}^{-1}}$ & $\frac{\Delta_{\mathrm{int}} H_{\mathrm{m}}^{\infty}}{\mathrm{kJ} \cdot \mathrm{mol}^{-1}}$ & $\frac{\Delta_{\mathrm{cav}} H}{\mathrm{~kJ} \cdot \mathrm{mol}}$ & $\frac{\Delta_{\mathrm{int}} H_{\mathrm{m}}^{\infty}}{\mathrm{kJ} \cdot \mathrm{mol}^{-1}}$ \\
\hline $\mathrm{W}$ & 7.1 & -38.1 & 7.5 & -75.9 & 7.8 & -87.9 & 9.0 & -172.5 \\
FMD & 28.9 & -52.9 & 30.2 & -85.5 & 31.5 & -100.0 & 36.5 & -201.2 \\
DMSO & 35.9 & -55.8 & 37.5 & -91.7 & 39.2 & -107.1 & 45.3 & -213.2 \\
\hline
\end{tabular}

\section{Discussion}

As pointed out in previous work ${ }^{(17)}$ the results obtained for the enthalpy of solvation of the systems studied in this work are difficult to correlate with the properties of the solutes and solvents involved. Unlike the enthalpy of solvation, the enthalpy of solute/solvent interaction provides a deeper insight into solvation as can be seen from what follows.

Cyclohexane in any solvent gives rise to negative values for $\Delta_{\mathrm{int}} H_{\mathrm{m}}^{\infty}$. As this solute contains six $\mathrm{CH}_{2}$ groups it is possible to estimate the enthalpy of interaction between one of these groups and the solvent. The results thus obtained are as follows: $-6.3 \mathrm{~kJ} \cdot \mathrm{mol}^{-1}$ in $\mathrm{W} ;-8.8 \mathrm{~kJ} \cdot \mathrm{mol}^{-1}$ in FMD; and $-9.3 \mathrm{~kJ} \cdot \mathrm{mol}^{-1}$ in DMSO.

The contribution of the $\mathrm{OH}$ group to $\Delta_{\mathrm{int}} H_{\mathrm{m}}^{\infty}$ can be estimated from the difference between the results obtained for the hydroxylated solutes and the literature data of cyclohexane. In doing so, the values calculated for the polar part of the solutes are probably underestimated since the interaction of $\mathrm{CH}$ is considered similar to that of $\mathrm{CH}_{2}$; it should be somewhat smaller. For cyclohexanol, the values obtained for $\mathrm{OH}$ are: $-38 \mathrm{~kJ} \cdot \mathrm{mol}^{-1}$ in $\mathrm{W} ;-33 \mathrm{~kJ} \cdot \mathrm{mol}^{-1}$ in FMD; and $-36 \mathrm{~kJ} \cdot \mathrm{mol}^{-1}$ in DMSO.

The values calculated for the interaction of the polar part of cyclohexanediol and inositol per mole of $\mathrm{OH}$ groups are not as negative as those observed for the monoalcohol. Following the procedure used for cyclohexanol, a contribution of about $-25 \mathrm{~kJ} \cdot \mathrm{mol}^{-1}$ for $\Delta_{\mathrm{int}} H_{\mathrm{m}}^{\infty}$ in all systems is obtained.

The decrease of solvation when the $\mathrm{OH}$ groups are attached to adjacent carbon atoms may be due to several reasons. A possible explanation may lie in the existence of intramolecular hydrogen bonding described for polyols in the gas phase, or dissolved in inert solvents. In fact cis-1,2-cyclohexanediol $^{(25,26)}$ and myo-inositol ${ }^{(27)}$ exhibit intramolecular hydrogen bonds which require some energy for disruption in polar media.

Theoretical calculations provide evidence for the solvation of diols in which one water molecule is hydrogen-bonded to two neighbouring $\mathrm{OH}$ groups of the solute. ${ }^{(28,29)}$ The proximity of the hydroxyl groups would also give rise to a decrease in the solvation by not allowing the full solvation sphere of the groups.

An important point to be considered in solvation is the behaviour of water towards nonpolar solutes. It has been observed that non-polar molecules give rise to unexpected results for the thermodynamic properties of solvation, namely large negative values for the entropy of solvation, and high partial molar heat capacity of the solute. 
Since the pioneering work of Frank and Evans in $1945^{(30)}$ the thermodynamic properties of non-polar substances have been often interpreted by assuming the formation of a layer of highly ordered water around the solute. Several molecular models have been proposed for describing such a structure. ${ }^{(30-32)}$ Nevertheless, some results obtained for these solutions cannot be interpreted in light of this theory. ${ }^{(33-36)}$ Other interpretations of the thermodynamic data have been proposed, and arguments against a structural water enhancement induced by non-polar solutes were presented. ${ }^{(37-44)}$

Despite the increasing power of computers for the simulation of the thermodynamic properties of solutions and the calculation of integral equations for hydration, ${ }^{(45)}$ not much light has been shed on the mechanism of hydration of this type of solute. At present, even the fundamental question of the degree of organization of water around a non-polar solute during hydration is awaiting an answer.

The decomposition of a solvation property into hard and soft contributions has shown that the results observed for water suggest the dominance of the former component. ${ }^{(46)}$ The present paper reinforces this thesis.

As can be seen from table $1, \Delta_{\text {solv }} H_{\mathrm{m}}^{\infty}$ for cyclohexane, cyclohexanol, and cyclohexanediol in water are more negative than in the other solvents. This cannot be understood from the view point of the molecular properties of the solvents, namely dipole moment and polarizability. Indeed, water, among the solvents studied in this work, has the smallest values for these properties, and therefore less negative solvation values should be expected.

By taking $\Delta_{\mathrm{int}} H_{\mathrm{m}}^{\infty}$ instead of $\Delta_{\text {solv }} H_{\mathrm{m}}^{\infty}$, a much clearer picture of the solvation phenomenon emerges. First, the "anomalous" behaviour of water given by $\Delta_{\text {solv }} H_{\mathrm{m}}^{\infty}$ disappears. $\Delta_{\mathrm{int}} H_{\mathrm{m}}^{\infty}$ follows an order determined by the dipole moment and polarizability of solutes and solvents. For any solute $\Delta_{\mathrm{int}} H_{\mathrm{m}}^{\infty}$ is less negative in water than in any other solvent.

By examining the values obtained for $\Delta_{\mathrm{cav}} H$ and $\Delta_{\mathrm{int}} H_{\mathrm{m}}^{\infty}$ given in table 3, one comes to the conclusion that the difference between water and the organic solvents lies in $\Delta_{\text {cav }} H$. This quantity is smaller for water than for the other solvents because of a smaller molecular diameter and a lower density number. The differences between the solvent in the enthalpy of cavity formation can be appreciated from the values obtained for the $y$ parameter which are as follows: 0.36 for water, 0.48 for formamide, and 0.52 for dimethylsulphoxide.

Owing to a weak solute/solvent interaction of the non-polar solutes in all solvents the difference in $\Delta_{\mathrm{int}} H_{\mathrm{m}}^{\infty}$ between solvents is rather small. Although $\Delta_{\mathrm{int}} H_{\mathrm{m}}^{\infty}$ of a nonpolar solute in water is less negative than in other solvents, $\Delta_{\text {cav }} H$ for water is much smaller than the corresponding values for organic solvents. Hence $\Delta_{\text {solv }} H_{\mathrm{m}}^{\infty}$ for this type of solute is more negative in water than in the other solvents. For instance, $\Delta_{\mathrm{int}} H_{\mathrm{m}}^{\infty}$ of cyclohexane in formamide is $15 \mathrm{~kJ} \cdot \mathrm{mol}^{-1}$ more negative than in water but as the difference between $\Delta_{\text {cav }} H$ of the two solvents is $22 \mathrm{~kJ} \cdot \mathrm{mol}^{-1}, \Delta_{\text {solv }} H_{\mathrm{m}}^{\infty}$ of this solute in water is $7.3 \mathrm{~kJ} \cdot \mathrm{mol}^{-1}$ more negative than in formamide. That is, for non-polar solutes, which in the present work include cyclohexanol and cyclohexanediol, besides cyclohexane the differences of $\Delta_{\mathrm{int}} H_{\mathrm{m}}^{\infty}$ between water and other solvents are overcome by the differences of $\Delta_{\text {cav }} H$.

As the polar part of the solute becomes dominant, $\Delta_{\mathrm{int}} H_{\mathrm{m}}^{\infty}$ decreases in all solvents, with the decrease being more pronounced in non-aqueous than in aqueous media. The 
differences in $\Delta_{\text {int }} H_{\mathrm{m}}^{\infty}$ between the solvents exceed those found for $\Delta_{\text {cav }} H$, and the ordering of the solutes becomes in qualitative terms the same as that given either by $\Delta_{\text {solv }} H_{\mathrm{m}}^{\infty}$ or by $\Delta_{\mathrm{int}} H_{\mathrm{m}}^{\infty}$. Of course, $\Delta_{\mathrm{cav}} H$ affects the solvation of all systems but its effect on the $\Delta_{\text {solv }} H_{\mathrm{m}}^{\infty}$ value depends on the relative values of $\Delta_{\text {int }} H_{\mathrm{m}}^{\infty}$ and $\Delta_{\text {cav }} H$.

A few conclusions can be drawn from this work which do not support the existence of a structural enhancement of water induced by non-polar solutes. Thermodynamic properties are still the most reliable methods for obtaining information on solvation. Moreover, the quantitative determination for solute/solvent interaction is a step forward towards a chemical interpretation of the thermodynamic properties of solvation. This can be achieved by using the scaled particle theory for an independent calculation of the contribution due to cavity formation in the solvent to accommodate the solute. For the enthalpy of solvation and for the systems studied here this theory gives a reasonable approximation. From the data presented no evidence was found for the existence of a layer of more ordered water around the solute.

\section{REFERENCES}

1. Sisskind, B.; Kasarnowsky, I. Z. Anorg. Allgem. Chem. 1933, 214, 385-395.

2. Eley, D. D. Trans. Faraday Soc. 1939, 35, 1281-1293, 1421-1432.

3. Reiss, H.; Frisch, H. L.; Lebowitz, J. L. J. Chem. Phys. 1959, 31, 369-380.

4. Reiss, H.; Casberg, R. V. J. Chem. Phys. 1974, 61, 1107-1114.

5. Pierotti, R. A. J. Phys. Chem. 1963, 67, 1840-1845.

6. Pierotti, R. A. J. Phys. Chem. 1965, 69, 281-288.

7. Pierotti, R. A. Chem. Rev. 1976, 76, 717-726.

8. Ben-Naim, A.; Friedman, H. L. J. Phys. Chem. 1967, 71, 448-449.

9. Stillinger, F. H. J. Solution Chem. 1973, 2, 141-158

10. DeVoe, H. J. Am. Chem. Soc. 1976, 98, 1724-1729.

11. Lucas, M. J. Phys. Chem. 1976, 80, 359-362.

12. Neumann, H. M. J. Solution Chem. 1977, 6, 33-38.

13. Morel-Desrosiers, N.; Morel, J.-P. Can. J. Chem. 1981, 59, 1-7.

14. Irisa, N.; Nakamura, H.; Irata, M. Chem. Phys. Lett. 1993, 207, 430-435.

15. Pohorille, A.; Pratt, L. R. J. Am. Chem. Soc. 1990, 112, 5066-5074.

16. Postma, J. P. M.; Berendsen, H. J. C.; Haak, J. R. Faraday Symp. Chem. Soc. 1982, 17, 55-67.

17. Costa, F. S.; Eusébio, M. E.; Redinha, J. S.; Leitão, M. L. P. J. Chem. Thermodynamics 1999, 31, 895-903.

18. a) Gill, S. J.; Nichols, F. N.; Wadsö, I. J. Chem. Thermodynamics 1976, 8, 445-452. b) Berling, D.; Olofsson, G. J. Solution Chem. 1994, 23, 911-923. c) Krishnan, C. V.; Friedman, H. L. J. Phys. Chem. 1969, 73, 1572-1580.

19. de Ligny, C. L.; van der Veen, N. G. Recueil Trav. Chim. Pays Bas 1971, 90, 984-1001.

20. de Ligny, C. L.; van der Veen, N. G. J. Solution Chem. 1975, 4, 841-851.

21. de Ligny, C. L.; van der Veen, N. G. Chem. Eng. Sci. 1972, 27, 391-401.

22. Wilhelm, E.; Battino, R. J. Chem. Phys. 1971, 55, 4012-4017.

23. Marcus, Y. Ion Solvation. John Wiley \& Sons: Chichester. 1985, Chap. 6.

24. Bondi, A. J. Phys. Chem. 1964, 68, 441-451.

25. Cole, A. R. H.; Jefferies, P. R. J. Chem. Soc. 1956, 4391-4397.

26. Allinger, N. L.; Rahman, M.; Lii, J.-H. J. Am. Chem. Soc. 1990, 112, 8293-8307.

27. Liang, C.; Ewig, C. S.; Stouch, T. R.; Hagler, A. T. J. Am. Chem. Soc. 1994, 116, 3904-3911.

28. Friedemann, R.; Jabs, A. J. Mol. Struct. (Theochem.) 1993, 283, 191-197.

29. Masella, M.; Flament, J.-P. Bull. Soc. Chim. Fr. 1997, 134, 439-449.

30. Frank, H. S.; Evans, M. W. J. Chem. Phys. 1945, 13, 504-532. 
31. Nemethy, G.; Sheraga, H. A. J. Phys. Chem. 1962, 36, 3401-3417.

32. Zichi, D. A.; Rossky, P. J. J. Chem. Phys. 1985, 83, 797-808.

33. Clifford, J.; Pethica, B. A. Trans. Faraday Soc. 1964, 60, 1483-1490

34. Bhanumathi, R.; Vijayalakshamma, S. K. J. Phys. Chem. 1986, 90, 4666-4669.

35. Muller, N. J. Solution Chem. 1988, 17, 661-672.

36. Finney, J. L.; Soper, A. K. Chem. Soc. Rev. 1994, 23, 1-10.

37. Aranow, R. H.; Witten, L. J. Phys. Chem. 1960, 64, 1643-1648.

38. Holtzer, A.; Emerson, M. F. J. Phys. Chem. 1969, 73, 26-33.

39. Howarth, O. W. J. Chem. Soc., Faraday Trans. I 1975, 71, 2303-2309.

40. Osinga, M. J. Am. Chem. Soc. 1979, 101, 1621-1622.

41. Cramer III, R. D. J. Am. Chem. Soc. 1977, 99, 5408-5412.

42. Wertz, D. H. J. Am. Chem. Soc. 1980, 102, 5316-5322.

43. Dec, S. F.; Gill, S. J. J. Solution Chem. 1985, 14, 827-836.

44. Besseling, N. A. M.; Lyklema, J. Pure Appl. Chem. 1995, 67, 881-888.

45. Ohmine, I.; Tanaka, H. Chem. Rev. 1993, 93, 2545-2566.

46. Ben-Naim, A. Water and Aqueous Solutions. Plenum Press: New York. 1974, Chap. 7.

(Received 8 June 1998; in final form 22 July 1999)

WA98/030 